\title{
WIENER ALGEBRA FOR THE QUATERNIONS
}

\author{
DANIEL ALPAY, FABRIZIO COLOMBO, DAVID P. KIMSEY, AND IRENE SABADINI
}

\begin{abstract}
We define and study the counterpart of the Wiener algebra in the quaternionic setting, both for the discrete and continuous case. We prove a Wiener-Lévy type theorem and a factorization theorem. We give applications to Toeplitz and Wiener-Hopf operators.
\end{abstract}

\section{INTRODUCTION AND PRELIMINARIES}

The Wiener algebra $\mathcal{W}^{n \times n}$ consists of the functions of the form $f(t)=\sum_{u \in \mathbb{Z}} f_{u} e^{i u t}$ where $f_{u} \in \mathbb{C}^{n \times n}$ and $\sum_{u \in \mathbb{Z}}\left\|f_{u}\right\|<\infty$, where $\|\cdot\|$ denotes the operator norm. The space $\mathcal{W}^{n \times n}$ with pointwise multiplication and the norm defined above is a Banach algebra. We denote by $\mathcal{W}_{+}^{n \times n}$ (resp. $\mathcal{W}_{-}^{n \times n}$ ) the subalgebra of functions $f \in \mathcal{W}^{n \times n}$ for which $f_{u}=0$ for $u<0$ (resp. $f_{u}=0$ for $u>0$ ). When $n=1$ we will denote the Wiener algebra by $\mathcal{W}$.

The Wiener algebra is important both in harmonic and complex analysis. The celebrated Wiener-Lévy theorem characterizes the invertible elements of $\mathcal{W}^{n \times n}$ : an element is invertible in $\mathcal{W}^{n \times n}$ if and only if it is pointwise invertible (in $\mathbb{C}^{n \times n}$ ). For $n=1$, this result was first proved by Wiener, see [13]. It is also a consequence of the theory of commutative Banach algebras, see [12, and for the case of coefficients in a normed ring see 3. In this paper we discuss how to generalize the Wiener algebra to the quaternionic setting, and we prove an analogue of the Wiener-Lévy theorem and the Wiener-Hopf factorization. The fact that the quaternions are a skew field requires tools different from those used in the complex case. Here we consider the case of quaternionic-valued functions, while the case of quaternionic matrix-valued functions will require other methods.

In the sequel, by $\mathbb{H}$ we denote the real algebra of quaternions. A quaternion $p$ is an element of the form $p=x_{0}+x_{1} e_{1}+x_{2} e_{2}+x_{3} e_{3}$ where $e_{1}^{2}=e_{2}^{2}=e_{1} e_{2} e_{3}=-1$. We will denote by $\mathbb{S}$ the sphere of unitary purely imaginary quaternions. Any element $i \in \mathbb{S}$ is such that $i^{2}=-1$ and any two orthogonal elements $i, j \in \mathbb{S}$ form a new basis $i, j, i j$ of $\mathbb{H}$. Given a quaternion $p_{0}$, it determines a sphere $\left[p_{0}\right]$ consisting of all the points of the form $q^{-1} p_{0} q$ for $q \neq 0$.

Date: November 19, 2014.

2010 Mathematics Subject Classification. 13J05,47S,47B35.

Key words and phrases. quaternions, Wiener algebras, Toeplitz operators, Wiener-Hopf operators.

D. Alpay thanks the Earl Katz family for endowing the chair which supported his research. D.P. Kimsey gratefully acknowledges the support of a Kreitman postdoctoral fellowship. F. Colombo and I. Sabadini acknowledge the Center for Advanced Studies of the Mathematical Department of the Ben-Gurion University of the Negev for the support and the kind hospitality during the period in which part of this paper has been written. 
A quaternionic valued function $f(p)$ belongs to the (discrete) quaternionic Wiener algebra $\mathcal{W}_{\mathbb{H}}$ if it is of the form $\sum_{u \in \mathbb{Z}} p^{u} f_{u}$ where $\sum_{u \in \mathbb{Z}}\left|f_{u}\right|<\infty$. The sum of two elements in $\mathcal{W}_{\mathbb{H}}$ is defined in the natural way while their product, denoted by $\star$, is obtained by taking the convolution of the coefficients (see 8 and Section 2). In order to relate the $\star$-product of two elements in $\mathcal{W}_{\mathbb{H}}$ with the pointwise product it is necessary to introduce a suitable map, that we denote by $\omega$ which allows to associate to the values of a quaternionic function to a $2 \times 2$ matrix with complex entries. This map is suitably defined by using the more classical map $\chi$ which, once that one fixes an imaginary unit $i$ and another imaginary unit, say $j$, orthogonal to $i$, associates to $p=z+w j$ the matrix

$$
\left(\begin{array}{cc}
z & w \\
-\bar{w} & \bar{z}
\end{array}\right)
$$

The new map $\omega$ is crucial to characterize the invertible elements in the Wiener algebra, see Theorem 2.5. It is interesting to note a specific feature of the class of functions we are considering: one of the equivalent conditions in this result is required only on one specific complex plane (so on a subset of $\mathbb{H}$ ) while another condition is required to hold globally. A similar result can be proved also for the subalgebra $\mathcal{W}_{\mathbb{H},+}$ (resp. $\mathcal{W}_{\mathbb{H},-}$ ) of elements such that $f_{u}=0$ for $u<0$ (resp. $u>0$ ). The definition of quaternionic Wiener algebra in the continuous case is somewhat more complicated, see Section 4. Also in this case we can define in a suitable way a map, still denoted by $\omega$, which allows to translate the image through $\omega$ of the $\star-$ product of two elements in the algebra with the pointwise product of the images of the two elements. Also in this case, we characterize the invertibility in the algebra. In this case, see Theorem 4.3 we still have equivalent conditions, and one condition holds on one specific complex plane if and only if it holds for any complex plane. Unlike the discrete case, the notions of positivity and of factorization depends on the choice of an imaginary unit.

Our study shows that the Wiener algebra in the quaternionic case not only requires suitable tools but also shows features which do not appear in the complex case.

The paper consists of four sections besides the introduction. Sections 2 and 3 are devoted to the discrete case, respectively the study of the discrete Wiener algebra and Toeplitz operators. Sections 4 and 5 deal with the continuous case. Specifically, in Section 4 we study the continuous Wiener algebra and in Section 5 we recall results on the quaternionic Hardy space of the half space and give an application to Wiener-Hopf operators.

\section{The Wiener Algebra}

In this section we define the counterpart of the Wiener algebra in the quaternionic setting.

Definition 2.1. We denote by $\mathcal{W}_{\mathbb{H}}$ the set of functions of the form

$$
f(p)=\sum_{u \in \mathbb{Z}} p^{u} f_{u}
$$

where the $f_{u}, u \in \mathbb{Z}$, are quaternions such that

$$
\sum_{u \in \mathbb{Z}}\left|f_{u}\right|<\infty
$$


Remark 2.2. Quaternionic power series have been considered, though in a different context, namely in the framework of slice hyperholomorphic functions, in [5] and [10.

The elements of $\mathcal{W}_{\mathbb{H}}$ are continuous functions on the closed unit ball $\overline{\mathbb{B}}$. The set $\mathcal{W}_{\mathbb{H}}$ be endowed with the multiplication (see [8])

$$
(f \star g)(p)=\sum_{u \in \mathbb{Z}} p^{u}\left(\sum_{k \in \mathbb{Z}} f_{u-k} g_{k}\right) .
$$

By extending the analogous result for polynomials with coefficients in a non commutative ring to power series (see [11) we have the formula

$$
(f \star g)(p)=f(p) g\left(f(p)^{-1} p f(p)\right)
$$

which holds for $f(p) \neq 0$ while for $f(p)=0$ we have $(f \star g)(p)=0$.

Proposition 2.3. $\mathcal{W}_{\mathbb{H}}$ endowed with the $\star$-multiplication is a real algebra.

Proof. The claim follows from

$$
\sum_{u \in \mathbb{Z}}\left|\sum_{k \in \mathbb{Z}} f_{u-k} g_{k}\right| \leq \sum_{u \in \mathbb{Z}} \sum_{k \in \mathbb{Z}}\left|f_{u-k}\right| \cdot\left|g_{k}\right|=\left(\sum_{u \in \mathbb{Z}}\left|f_{u}\right|\right)\left(\sum_{k \in \mathbb{Z}}\left|g_{k}\right|\right) .
$$

We will call $\mathcal{W}_{\mathbb{H}}$ the quaternionic Wiener algebra.

Let $i, j \in \mathbb{S}$ be orthogonal (that is, such that $i j+j i=0$ ). Then any quaternion $p$ can be written as $p=z+w j$, where $z, w$ complex numbers belonging to the complex plane $\mathbb{C}_{i}=\{x+i y: x, y \in \mathbb{R}\}$. In the sequel, we will sometimes write $\mathbb{C}$, for short. We have an injective homomorphism of rings $\chi=\chi_{i, j}: \mathbb{H} \rightarrow \mathbb{C}^{2 \times 2}$ defined by

$$
\chi(p)=\left(\begin{array}{cc}
z & w \\
-\bar{w} & \bar{z}
\end{array}\right) .
$$

Let $f$ be as in 2.1). Then, restricting $p=e^{i t}$ to the unit circle, writing $f\left(e^{i t}\right)=$ $a\left(e^{i t}\right)+b\left(e^{i t}\right) j$ we obtain

$$
\chi\left(f\left(e^{i t}\right)\right)=\left(\begin{array}{cc}
a\left(e^{i t}\right) & \frac{b\left(e^{i t}\right)}{a\left(e^{i t}\right)}
\end{array}\right),
$$

where the functions

$$
a\left(e^{i t}\right)=\sum_{u \in \mathbb{Z}} e^{i u t} a_{u} \quad \text { and } \quad b\left(e^{i t}\right)=\sum_{u \in \mathbb{Z}} e^{i u t} b_{u}
$$

belong to the classical Wiener algebra, denoted by $\mathcal{W}:=\mathcal{W}^{1 \times 1}$. Thus,

$$
\chi\left(f\left(e^{i t}\right)\right) \in \mathcal{W}^{2 \times 2} .
$$

It is easy to verify that the map $\chi$ is not multiplicative with respect to the $\star$-product, in fact in general

$$
\chi\left((f \star g)\left(e^{i t}\right)\right) \neq \chi\left((f)\left(e^{i t}\right)\right) \chi\left((g)\left(e^{i t}\right)\right) .
$$

We now introduce another map depending on the choice of two orthogonal imaginary units $i, j \in \mathbb{S}$ and denoted for simplicity by $\omega:=\omega_{i, j}$, which acts from the values of functions (belonging to a given set of functions) to the set of $2 \times 2$ matrices 
whose elements are complex-valued functions when restricted to the complex plane $\mathbb{C}$ and it is defined by

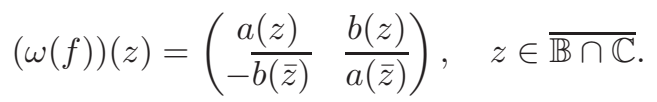

Note that the definition of $\omega$ makes sense if one substitutes $z$ with any $p \in \mathbb{B}$.

Lemma 2.4. Let $f, g \in \mathcal{W}_{\mathbb{H}}$. Then it holds that

$$
(\omega(f \star g))(z)=(\omega(f))(z)(\omega(g))(z), \quad z \in \overline{\mathbb{B} \cap \mathbb{C}} .
$$

Proof. Let $f(p)=p^{n} a$ and $g(p)=p^{m} b$ for $n, m \in \mathbb{Z}$ and $a, b \in \mathbb{H}$. Then,

$$
(f \star g)(p)=p^{n+m} a b,
$$

and we have

$$
(\omega(f))\left(z^{n}\right)=z^{n} \chi(a)
$$

and similarly

$$
(\omega(g))(z)=z^{m} \chi(b), \quad(\omega(f \star g))(z)=z^{n+m} \chi(a b) .
$$

Hence

$$
(\omega(f \star g))(z)=z^{n+m} \chi(a b)=z^{n+m} \chi(a) \chi(b)=(\omega(f))(z)(\omega(g))(z) .
$$

More generally, if $f(p)=\sum_{n \in \mathbb{Z}} p^{n} a_{n} \in \mathcal{W}_{\mathbb{H}}$, then

$$
(\omega(f))(z)=\sum_{n \in \mathbb{Z}} z^{n} \chi\left(a_{n}\right) \in \mathcal{W}^{2 \times 2} .
$$

Moreover, if $g(p)=\sum_{n \in \mathbb{Z}} p^{n} b_{n} \in \mathcal{W}_{\mathbb{H}}$, then

$$
\begin{aligned}
((\omega(f))(z))((\omega(g))(z)) & =\left(\sum_{n \in \mathbb{Z}} z^{n} \chi\left(a_{n}\right)\right)\left(\sum_{n \in \mathbb{Z}} z^{n} \chi\left(b_{n}\right)\right) \\
& =\sum_{u \in \mathbb{Z}} z^{u}\left(\sum_{n+m=u} \chi\left(a_{n}\right) \chi\left(b_{m}\right)\right) \\
& =\sum_{u \in \mathbb{Z}} z^{u} \chi\left(\sum_{n+m=u} a_{n} b_{m}\right) \\
& =(\omega(f \star g))(z) .
\end{aligned}
$$

Theorem 2.5. Let $f \in \mathcal{W}_{\mathbb{H}}$. The following are equivalent:

(i) $f$ is invertible in $\mathcal{W}_{\mathbb{H}}$;

(ii) Let $i$ be any fixed element in $\mathbb{S}$, then $(\operatorname{det} \omega(f))(z) \neq 0$ for all $z \in \partial \mathbb{B} \cap \mathbb{C}_{i}$;

(iii) The function $f$ does not vanish on $\partial \mathbb{B}$.

Proof. The implication (i) $\Longrightarrow$ (ii) is a direct consequence of (2.7): if there exists $g \in \mathcal{W}_{\mathbb{H}}$ such that $f \star g=1$ then

$$
I_{2}=\left(\begin{array}{ll}
1 & 0 \\
0 & 1
\end{array}\right)=\left(\omega(f)\left(e^{i t}\right)\right)\left(\omega(g)\left(e^{i t}\right)\right)
$$

and so $\omega(f)\left(e^{i t}\right)$ is invertible for every $t \in[0,2 \pi)$.

To prove the converse we first note that $\omega(f)(z) \in \mathcal{W}^{2 \times 2}$. By the classical matricial Wiener-Lévy theorem the condition $\operatorname{det} \omega(f)\left(e^{i t}\right) \neq 0$ for all $t \in[0,2 \pi)$ implies that 
$\omega(f)$ is invertible in $\mathcal{W}^{2 \times 2}$. Let $G \in \mathcal{W}^{2 \times 2}$ be such that $\omega(f) G=I_{2}$. By the formula for the inverse of a $2 \times 2$ matrix we have that $G$ is of the form

$$
G\left(e^{i t}\right)=\left(\frac{c\left(e^{i t}\right)}{-d\left(e^{-i t}\right)} \frac{d\left(e^{i t}\right)}{c\left(e^{-i t}\right)}\right),
$$

where $c\left(e^{-i t}\right)=\overline{a\left(e^{-i t}\right)} /(\operatorname{det} \omega(f))\left(e^{i t}\right)$ and $d\left(e^{-i t}\right)=-b\left(e^{i t}\right) /(\operatorname{det} \omega(f))\left(e^{i t}\right)$ so they belong to $\mathcal{W}$. Thus we can write

$$
c\left(e^{i t}\right)=\sum_{n \in \mathbb{Z}} e^{i n t} c_{n} \quad \text { and } \quad d\left(e^{i t}\right)=\sum_{n \in \mathbb{Z}} e^{i n t} d_{n} .
$$

If we set

$$
g\left(e^{i t}\right)=\sum_{n \in \mathbb{Z}} e^{i n t}\left(c_{n}+d_{n} j\right),
$$

then $g \in \mathcal{W}_{\mathbb{H}}$. Since $\omega(f) \omega(g)=1$ on the unit circle, if $f(p)=\sum_{n \in \mathbb{Z}} p^{n} f_{n}$ and $g(p)=\sum_{n \in \mathbb{Z}} p^{n} g_{n}$, we have

$$
\chi\left(\sum_{n+m=u} f_{n} g_{m}\right)=\left\{\begin{array}{l}
0 \text { if } u \neq 0, \\
1 \text { if } u=0 .
\end{array}\right.
$$

Hence $(f \star g)\left(e^{i t}\right)=1$ and thus $f \star g=1$ everywhere, since the latter is uniquely determined by its values on the unit circle.

We now show that (ii) and (iii) are equivalent. An easy computation, using the notation in (2.6), shows that for some fixed $i \in \mathbb{S}$

$$
(\operatorname{det} \omega(f))(z)=a(z) \overline{a(\bar{z})}+b(z) \overline{b(\bar{z})}=\sum_{u \in \mathbb{Z}} z^{u}\left(\sum_{k \in \mathbb{Z}} a_{u-k} \overline{a_{k}}+b_{u-k} \overline{b_{k}}\right),
$$

where the functions $a$ and $b$ are defined by (2.4).

Given $f(p)=\sum_{u \in \mathbb{Z}} p^{u} f_{u} \in \mathcal{W}_{\mathbb{H}}$ we define the function $f^{c}(p)=\sum_{u \in \mathbb{Z}} p^{u} \overline{f_{u}}$ which still belongs to $\mathcal{W}_{\mathbb{H}}$. The function $\left(f \star f^{c}\right)(p)=\sum_{u \in \mathbb{Z}} p^{u}\left(\sum_{k \in \mathbb{Z}} f_{u-k} \overline{f_{k}}\right)$ has real coefficients, thus the zeros of its restriction $\left(f \star f^{c}\right)_{\mid \mathbb{C}}(z)$ to $\mathbb{C}$ has zeros which are real points and/or complex conjugate points. By the validity of the representation formula for power series, see [5], it follows that $\left(f \star f^{c}\right)(p)$ has zero set consisting of real points and/or spheres. By formula (2.2) we have that $\left(f \star f^{c}\right)\left(p_{0}\right)=0$ implies $f\left(p_{0}\right)=0$ or $f\left(p_{0}\right) \neq 0$ and $f^{c}\left(f\left(p_{0}\right)^{-1} p_{0} f\left(p_{0}\right)\right)=0$. Note that the element $f\left(p_{0}\right)^{-1} p_{0} f\left(p_{0}\right)$ belongs to $\left[p_{0}\right]$. However the zeros of $f^{c}$ on $\left[p_{0}\right]$ are in one-to-one correspondence with the zeros of $f$ on $\left[p_{0}\right]$, in fact

$$
f(x+i y)=\sum_{u \in \mathbb{Z}}(x+i y)^{u} f_{u}=\sum_{u \in \mathbb{Z}}\left(s_{u}(x, y)+i t_{u}(x, y)\right) f_{u}=s(x, y)+i t(x, y)
$$

where the functions $s_{u}, t_{u}$ are real valued. Similarly,

$$
f^{c}(x+i y)=\sum_{u \in \mathbb{Z}}(x+i y)^{u} \bar{f}_{u}=\sum_{u \in \mathbb{Z}}\left(s_{u}(x, y)+i t_{u}(x, y)\right) \bar{f}_{u}=\overline{s(x, y)}+\overline{i t(x, y)} .
$$

If $f$ vanishes at all points of $\left[p_{0}\right]$ then $s\left(x_{0}, y_{0}\right)=t\left(x_{0}, y_{0}\right)=0$ and $f^{c}$ vanishes at all points of $\left[p_{0}\right]$. If $p_{0}$ is the only zero of $f$ on $\left[p_{0}\right]$ then $t\left(x_{0}, y_{0}\right) \neq 0$ and an immediate computation shows that $f^{c}\left(x_{0}+\tilde{\iota} y_{0}\right)=0$ where $\tilde{\iota}=-\overline{t\left(x_{0}, y_{0}\right)} \overline{i t\left(x_{0}, y_{0}\right)}-1$. The converse follows in an analogous way, since $\left(f^{c}\right)^{c}=f$. Thus if $f^{c}$ has a zero 
belonging to the sphere $\left[p_{0}\right]$ also $f$ must have a zero belonging to the same sphere. Now we observe that since $f_{u}=a_{u}+b_{u} j$ and $\overline{f_{u}}=\overline{a_{u}}-b_{u} j$ it is immediate that

$$
\begin{aligned}
\left(f \star f^{c}\right)_{\mid \mathbb{C}}(z) & =\sum_{u \in \mathbb{Z}} p^{u}\left(\sum_{k \in \mathbb{Z}} f_{u-k} \overline{f_{k}}\right)=\sum_{u \in \mathbb{Z}} p^{u}\left(\sum_{k \in \mathbb{Z}}\left(a_{u-k}+b_{u-k} j\right)\left(\bar{a}_{k}-b_{k} j\right)\right) \\
& =\sum_{u \in \mathbb{Z}} p^{u}\left\{\sum_{k \in \mathbb{Z}}\left(a_{u-k} \bar{a}_{k}+b_{u-k} \bar{b}_{k}+\left(b_{u-k} a_{k}-a_{u-k} b_{k}\right) j\right)\right\}
\end{aligned}
$$

and so $(\operatorname{det} \omega(f))(z)=\left(f \star f^{c}\right)_{\mid \mathbb{C}}(z)$.

If (ii) holds then $f$ cannot have zeros on $\partial \mathbb{B}$ otherwise, if $f\left(p_{0}\right)=0$ we have that $f \star f^{c}$ vanishes on $\left[p_{0}\right]$ and in particular on $\left[p_{0}\right] \cap \mathbb{C}$ and so $\operatorname{det}(\omega(f))$ vanishes on $\partial \mathbb{B} \cap \mathbb{C}$. Conversely, if $f$ does not vanish on $\partial \mathbb{B}$ neither $f^{c}$ vanishes there and so $f \star f^{c}$ does not have zeros on $\partial \mathbb{B}$ and thus $\operatorname{det}(\omega(f))$ does not vanish on $\partial \mathbb{B} \cap \mathbb{C}$.

Remark 2.6. Note that condition (ii) holds on a fixed plane while condition (iii) refers to the whole boundary of $\mathbb{B}$. Consider, for example, $f(p)=p-j$. It is immediate that $f$ does not have any zero on the complex plane $\mathbb{C}_{i}$. However, $\operatorname{det}(\omega(f))\left(e^{ \pm i \pi}\right)=0$.

Definition 2.7. We denote by $\mathcal{W}_{\mathbb{H},+}\left(\operatorname{resp} . \mathcal{W}_{\mathbb{H},-}\right)$ the set of elements $f(p)=$ $\sum_{n \in \mathbb{N}} p^{n} f_{n} \in \mathcal{W}_{\mathbb{H}}$, for which $f_{n}=0$ for $n<0$ (resp. for $n>0$ ).

It is immediate that $\mathcal{W}_{\mathbb{H},+}$ and $\mathcal{W}_{\mathbb{H},-}$ are subalgebras of $\mathcal{W}_{\mathbb{H}}$. The question of invertibility of an element in $\mathcal{W}_{\mathbb{H},+}$ can be asked in $\mathcal{W}_{\mathbb{H},+}$ and in $\mathcal{W}_{\mathbb{H}}$. For instance, $f(p)=p$ is invertible in $\mathcal{W}_{\mathbb{H}}$, but not in $\mathcal{W}_{\mathbb{H},+}$.

Theorem 2.8. Let $f \in \mathcal{W}_{\mathbb{H},+}$. The following are equivalent:

(i) The function $f$ is invertible in $\mathcal{W}_{\mathbb{H},+}$;

(ii) Let $i$ be any fixed element in $\mathbb{S}$, then $(\operatorname{det} \omega(f))(z) \neq 0$ for all $z \in \overline{\mathbb{B} \cap \mathbb{C}_{i}}$;

(iii) The function $f$ does not vanish on $\overline{\mathbb{B}}$.

Proof. The proof is very similar to the proof of Theorem 2.5. We will only provide details for the implication (ii) $\Longrightarrow(\mathrm{i})$. We begin by noting that $\omega(f) \in \mathcal{W}_{+}^{2 \times 2}$. By the result for invertiblity of matrix-valued functions in the Wiener algebra $\mathcal{W}_{+}^{2 \times 2}$ (see, e.g., 9]), the condition $\operatorname{det}(\omega(f))(z) \neq 0$ for $z \in \overline{\mathbb{B} \cap \mathbb{C}}$ implies that $\omega(f)$ is invertible in $\mathcal{W}_{+}^{2 \times 2}$. Let $G \in \mathcal{W}_{+}^{2 \times 2}$ be so that $\omega(f) G=I_{2}$ on $\overline{\mathbb{B} \cap \mathbb{C}}$. Similar to the proof of Theorem 2.5 we get that

$$
G(z)=\left(\begin{array}{cc}
\frac{c(z)}{d(\bar{z})} & \frac{d(z)}{c(z)}
\end{array}\right),
$$

where $c(z)=\overline{a(\bar{z})} \operatorname{det}(\omega(f))(z)$ and $d(z)=-b(z) \operatorname{det}(\omega(f))(z)$. Thus, we may write $c(z)=\sum_{n=0}^{\infty} z^{n} c_{n}$ and $d(z)=\sum_{n=0}^{\infty} z^{n} d_{n}$. If we set $g(z)=\sum_{n=0}^{\infty} z^{n}\left(c_{n}+d_{n} j\right)$, then $g \in \mathcal{W}_{\mathbb{H},+}$. The rest of the argument is as in the proof of Theorem 2.5.

Definition 2.9. We say that $f \in \mathcal{W}_{\mathbb{H}}$ is strictly positive if

$$
\omega(f)\left(e^{i t}\right)>0, \quad t \in[0,2 \pi) .
$$

Theorem 2.10. $f \in \mathcal{W}_{\mathbb{H}}$ is strictly positive if and only if it can be written as $f=f_{+} \star f_{+}^{c}$, where $f_{+}$is an invertible element of $\mathcal{W}_{\mathbb{H},+}$. 
Proof. By the classical Wiener-Hopf theory, there is an element $A \in \mathcal{W}_{+}^{2 \times 2}$, unique up to a right multiplicative unitary constant, such that $A^{-1} \in \mathcal{W}_{+}^{2 \times 2}$ and

$$
\omega(f)\left(e^{i t}\right)=A\left(e^{i t}\right) A\left(e^{i t}\right)^{*} .
$$

Let

$$
J_{1}=\left(\begin{array}{cc}
0 & 1 \\
-1 & 0
\end{array}\right)
$$

A simple computation shows that

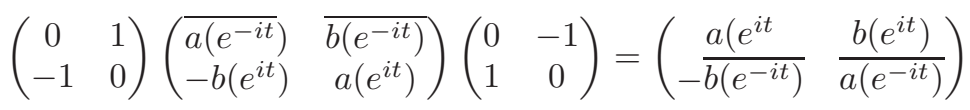

from which we deduce

$$
J_{1} \overline{\omega(f)\left(e^{-i t}\right)} J_{1}^{*}=\omega(f)\left(e^{i t}\right) .
$$

The function

$$
B\left(e^{i t}\right)=J_{1} \overline{A\left(e^{-i t}\right)} J_{1}^{*}
$$

is an invertible element of $\mathcal{W}_{+}^{2 \times 2}$. Thus, for a unitary constant $U$ we have

$$
A\left(e^{i t}\right)=J_{1} \overline{A\left(e^{-i t}\right)} J_{1}^{*} U .
$$

We have that $A(1)$ is invertible since $A\left(e^{i t}\right)$ is invertible in $\mathcal{W}_{+}^{2 \times 2}$. By replacing $A(z)$ by $A(z) A(1)^{*}\left(A(1) A(1)^{*}\right)^{-1 / 2}$ we can always choose $A(1)>0$, i.e., $A(1)$ is a positive definite matrix. This forces $U=I_{2}$. Indeed, let $A(1)=\left(\begin{array}{ll}a & \bar{c} \\ c & d\end{array}\right)$. Then

$$
A(1)=J_{1} \overline{A(1)} J_{1}^{*}
$$

and (2.9) leads to $U=I_{2}$. With $A(z)=\sum_{n=0}^{\infty} z^{n} A_{n}$ we have

$$
A_{n}=J_{1} \overline{A_{n}} J_{1}^{*} \text {, }
$$

and so

$$
A\left(e^{i t}\right)=\omega\left(a_{+}\right)\left(e^{i t}\right)
$$

where $a_{+}(p)=\sum_{n=0}^{\infty} p^{n} a_{n}$ and $\chi\left(a_{n}\right)=A_{n}$.

\section{TOEPLitz OPERATORS}

Let $\ell_{2}(\mathbb{H})$ denote the Hilbert space of all $\mathbb{H}$-valued sequences $a=\left(a_{n}\right)_{n=0}^{\infty}$ so that

$$
\|a\|_{\ell_{2}}^{2}=\sum_{n=0}^{\infty}\left|a_{n}\right|^{2}<\infty .
$$

The inner product for $a, b \in \ell_{2}(\mathbb{H})$ is given by

$$
\langle a, b\rangle_{\ell_{2}}=\sum_{n=0}^{\infty} \bar{b}_{n} a_{n} .
$$

Let $L_{2}(\partial \mathbb{B})$ denote the Hilbert space of all functions $f(p)=\sum_{n=-\infty}^{\infty} p^{n} f_{n}$ on $\partial \mathbb{B}$ so that

$$
\begin{aligned}
\|f\|_{L_{2}(\partial \mathbb{B})}^{2} & =\frac{1}{2 \pi} \int_{0}^{2 \pi}\left|f\left(e^{i \theta}\right)\right|^{2} d \theta \\
& =\sum_{n=-\infty}^{\infty}\left|f_{n}\right|^{2}<\infty .
\end{aligned}
$$


As in the complex case, elements of $L_{2}(\partial \mathbb{B})$ are defined almost everywhere on $\partial \mathbb{B}$. It is worth noting that (3.2) does not depend on $i \in \mathbb{S}$. Thus, any $i \in \mathbb{S}$ can be used in (3.1). The inner product for $L_{2}(\partial \mathbb{B})$ is given by

$$
\langle f, g\rangle_{\partial \mathbb{B}}=\frac{1}{2 \pi} \int_{0}^{2 \pi} \overline{g\left(e^{i \theta}\right)} f\left(e^{i \theta}\right) d \theta=\sum_{u=-\infty}^{\infty} \bar{g}_{u} f_{u} .
$$

We let $H_{2}(\partial \mathbb{B})$ and $H_{2}(\partial \mathbb{B})^{\perp}$ denote the set of functions $f \in L_{2}(\partial \mathbb{B})$ so that $f_{n}=0$ for $n<0$ and $f_{n}=0$ for $n \geq 0$, respectively.

Let $\mathfrak{p}$ and $\mathfrak{q}$ denote the orthogonal projections of $L_{2}(\partial \mathbb{B})$ onto $H_{2}(\partial \mathbb{B})$ and $L_{2}(\partial \mathbb{B})$ onto $\left(H_{2}(\partial \mathbb{B})\right)^{\perp}$, respectively. Note that $(\mathfrak{p}+\mathfrak{q}) f=f$ for $f \in L_{2}(\partial \mathbb{B})$. The operators $\mathfrak{p}$ and $\mathfrak{q}$ are given by the rules

$$
(\mathfrak{p} f)(p)=\sum_{n=0}^{\infty} p^{n} f_{n}
$$

and

$$
(\mathfrak{q} f)(p)=\sum_{n=-\infty}^{-1} p^{n} f_{n}
$$

respectively, where $f(p)=\sum_{n=-\infty}^{\infty} p^{n} f_{n}$. If $\varphi \in \mathcal{W}_{\mathbb{H}}$, then we let $M_{\varphi}$ denote the operator of multiplication on $\mathcal{W}_{\mathbb{H}}$ given by $\left(M_{\varphi} f\right)(p)=(\varphi \star f)(p)$. If $\varphi \in \mathcal{W}_{\mathbb{H}}$, then we define the Toeplitz operator $T_{\varphi}$ by the rule

$$
T_{\varphi} f=\mathfrak{p} M_{\varphi} f \quad \text { for } \quad f \in H_{2}(\partial \mathbb{B}) .
$$

The Toeplitz operator $T_{\varphi}$ can also be defined as an operator from $\ell_{2}(\mathbb{H}) \rightarrow \ell_{2}(\mathbb{H})$ given by

$$
T_{\varphi} \xi=\left(\begin{array}{cccc}
\varphi_{0} & \cdots & \varphi_{-n} & \cdots \\
\vdots & \ddots & \vdots & \ddots \\
\varphi_{n} & \cdots & \varphi_{0} & \cdots \\
\vdots & \ddots & \vdots & \ddots
\end{array}\right)\left(\begin{array}{c}
\xi_{0} \\
\vdots \\
\xi_{n} \\
\vdots
\end{array}\right) \quad \text { for } \quad \xi=\left(\begin{array}{c}
\xi_{0} \\
\vdots \\
\xi_{n} \\
\vdots
\end{array}\right) \in \ell_{2}(\mathbb{H}) \text {. }
$$

Theorem 3.1 and Corollary 3.2 are natural analogs of results established for Toeplitz operators in the classical setting (see [4).

Theorem 3.1. If $\varphi, \psi \in \mathcal{W}_{\mathbb{H}}$, then $T_{\varphi} T_{\psi}$ is Toeplitz if and only if $\varphi \in \mathcal{W}_{\mathbb{H},-}$ or $\psi \in \mathcal{W}_{\mathbb{H},+}$. In this case, $T_{\varphi} T_{\psi}=T_{\varphi \star \psi}$.

Proof. If $\psi \in \mathcal{W}_{\mathbb{H},+}$, then

$$
\begin{aligned}
T_{\varphi} T_{\psi} f & =\mathfrak{p} M_{\varphi} \mathfrak{p} M_{\psi} f \quad \text { for } \quad f \in H_{2}(\partial \mathbb{B}) \\
& =\mathfrak{p} M_{\varphi} M_{\psi} f \\
& =\mathfrak{p} M_{\varphi \star \psi} f \\
& =T_{\varphi \star \psi} f .
\end{aligned}
$$

If $\varphi \in \mathcal{W}_{\mathbb{H},-}$, then

$$
\begin{aligned}
T_{\varphi} T_{\psi} f & =\mathfrak{p} M_{\varphi} \mathfrak{p} M_{\psi} f \quad \text { for } \quad f \in H_{2}(\partial \mathbb{B}) \\
& =\mathfrak{p} M_{\varphi}(\mathfrak{p}+\mathfrak{q}) M_{\psi} f-\mathfrak{p} M_{\varphi} \mathfrak{q} M_{\psi} f \\
& =\mathfrak{p} M_{\varphi \star \psi} f-0 \\
& =T_{\varphi \star \psi} f .
\end{aligned}
$$


Conversely, if $T_{\varphi} T_{\psi}$ is Toeplitz, then

$$
T_{\varphi} T_{\psi}=T_{\varphi \star \psi} .
$$

Indeed, if there exists $g \in \mathcal{W}_{\mathbb{H}}$ so that

$$
\mathfrak{p} M_{\varphi} \mathfrak{p} M_{\psi} f=\mathfrak{p} M_{g} f \quad \text { for } \quad f \in H_{2}(\partial \mathbb{B}),
$$

then

$$
\mathfrak{p} M_{\varphi}(\mathfrak{p}+\mathfrak{q}-\mathfrak{q}) M_{\psi} f=\mathfrak{p} M_{g} f
$$

whence

$$
\mathfrak{p} M_{\varphi \star \psi-g} f=\mathfrak{p} M_{\varphi} \mathfrak{q} M_{\psi} f \quad \text { for } \quad f \in H_{2}(\partial \mathbb{B}) .
$$

Thus $g=\varphi \star \psi$. Finally, it follows from (3.5) that

$$
\mathfrak{p} M_{\varphi \star \psi} f-\mathfrak{p} M_{\varphi} \mathfrak{q} M_{\psi} f=\mathfrak{p} M_{\varphi \star \psi} f \quad \text { for } \quad f \in H_{2}(\partial \mathbb{B})
$$

i.e.

$$
\mathfrak{p} M_{\varphi} \mathfrak{q} M_{\psi} f=0 \quad \text { for } \quad f \in H_{2}(\partial \mathbb{B}) .
$$

It follows from (3.6) that $\varphi \in \mathcal{W}_{\mathbb{H},-}$ or $\psi \in \mathcal{W}_{\mathbb{H},+}$.

Corollary 3.2. If $\varphi, \psi \in \mathcal{W}_{\mathbb{H}}$, then $T_{\varphi} T_{\psi}=0$ if and only if $\varphi(p)=0$ for all $p \in \partial \mathbb{B}$ or $\phi(p)=0$ for all $p \in \partial \mathbb{B}$.

Proof. The converse statement is obvious. If $T_{\varphi} T_{\psi}=0=T_{0}$, then we may use (3.1) to deduce that $(\varphi \star \psi)(p)=0$ for all $p \in \partial \mathbb{B}$. But then we must have that $\varphi(p)=0$ for all $p \in \partial \mathbb{B}$ or $\psi(p)=0$ for all $p \in \partial \mathbb{B}$.

\section{The Wiener algebra in the COntinuous CASE}

Let $L_{1}(\mathbb{R}, \mathbb{H})$ denote the space of functions $f: \mathbb{R} \rightarrow \mathbb{H}$ so that

$$
\|f\|_{L_{1}(\mathbb{R}, \mathbb{H})}=\int_{\mathbb{R}}|f(u)| d u<\infty .
$$

We denote by $\mathcal{W}(\mathbb{R}, \mathbb{H})$ the set of functions of the form

$$
F_{1}(p)=c_{1}+\int_{\mathbb{R}} e^{p u} f_{1}(u) d u, \quad p \in \mathbb{R} \mathbb{S}=\{i t: t \in \mathbb{R} \text { and } i \in \mathbb{S}\},
$$

where $f_{1} \in L_{1}(\mathbb{R}, \mathbb{H})$ and $c_{1} \in \mathbb{H}$. The set $\mathcal{W}(\mathbb{R}, \mathbb{H})$ can be endowed with the multiplication

$$
\left(F_{1} \star F_{2}\right)(p)=c_{1} c_{2}+\int_{\mathbb{R}} e^{u p} c_{1} f_{2}(u) d u+\int_{\mathbb{R}} e^{u p} f_{1}(u) c_{2} d u+\int_{\mathbb{R}} e^{u p}\left(f_{1} \circ f_{2}\right)(u) d u
$$

where $f_{1} \circ f_{2}$ denotes the convolution of $f_{1}$ and $f_{2}$ and

$$
F_{2}(p)=c_{2}+\int_{\mathbb{R}} e^{u p} f_{2}(u) d u .
$$

If $F_{1}$ is as above, we also define

$$
\left\|F_{1}\right\|=\left|c_{1}\right|+\left\|f_{1}\right\|_{L_{1}(\mathbb{R}, \mathbb{H})} .
$$

We have:

Proposition 4.1. $\mathcal{W}(\mathbb{R}, \mathbb{H})$ endowed with the $\star$-multiplication is a real Banach algebra. 
Proof. The proof follows from

$$
\begin{aligned}
\left\|F_{1} \star F_{2}\right\| & \leq\left|c_{1} c_{2}\right|+\left\|f_{1} c_{2}+c_{1} f_{2}+f_{1} \circ f_{2}\right\|_{L_{1}(\mathbb{R}, \mathbb{H})} \\
& \leq\left|c_{1} c_{2}\right|+\left\|f_{1}\right\|_{L_{1}(\mathbb{R}, \mathbb{H})}\left|c_{2}\right|+\left|c_{1}\right|\left\|f_{2}\right\|_{L_{1}}+\left\|f_{1} \circ f_{2}\right\|_{L_{1}(\mathbb{R}, \mathbb{H})} \\
& =\left(\left|c_{1}\right|+\left\|f_{1}\right\|_{L_{1}(\mathbb{R}, \mathbb{H})}\right)\left(\left|c_{2}\right|+\left\|f_{2}\right\|_{L_{1}(\mathbb{R}, \mathbb{H})}\right)=\left\|F_{1}\right\|+\left\|F_{2}\right\| .
\end{aligned}
$$

The completeness follows just as in the complex case.

We now define a map $\omega=\omega_{i, j}$ depending on the choice of an imaginary unit $i \in \mathbb{S}$ and of a $j \in \mathbb{S}$ orthogonal to $i$. In what follows, we will omit to specify the imaginary units $i$ and $j$, but we keep the imaginary unit while writing the variables to make it explicit that we work on a specific complex plane (this leads to a different notation than in the classical case, where the imaginary unit is unique and can be understood). Then, by setting $p=i t$, we define

$$
\omega\left(F_{1}\right)(i t)=\chi\left(c_{1}\right)+\int_{\mathbb{R}} e^{i t u} \chi\left(f_{1}(u)\right) d u,
$$

where $\chi$ is defined as in (2.3), that is if $f_{1}(u)=a(u)+b(u) j$ then

$$
\chi\left(f_{1}(u)\right)=\left(\begin{array}{cc}
a(u) & \frac{b(u)}{a(u)}
\end{array}\right) .
$$

It is immediate that $a, b \in L_{1}(\mathbb{R}, \mathbb{C})$ and so $\omega\left(F_{1}\right)(i t)$ belongs to the continuous Wiener algebra $\mathcal{W}^{2 \times 2}(\mathbb{R})$ with values in $\mathbb{C}^{2 \times 2}$.

Lemma 4.2. Let $F_{1}, F_{2} \in \mathcal{W}(\mathbb{R}, \mathbb{H})$. Then

$$
\left(\omega\left(F_{1} \star F_{2}\right)\right)(i t)=\left(\omega\left(F_{1}\right)\right)(i t)\left(\omega\left(F_{2}\right)\right)(i t), \quad t \in \mathbb{R} .
$$

Proof. We have

$$
\begin{aligned}
\left(\omega\left(F_{1} \star F_{2}\right)\right)(i t) & =\chi\left(c_{1} c_{2}\right)+\int_{\mathbb{R}} e^{i t u} \chi\left(f_{1} c_{2}+c_{1} f_{2}+f_{1} \circ f_{2}\right)(u) d u \\
& =\chi\left(c_{1} c_{2}\right)+\int_{\mathbb{R}} e^{i t u}\left(\chi\left(f_{1} c_{2}\right)+\chi\left(c_{1} f_{2}\right)+\chi\left(f_{1} \circ f_{2}\right)\right)(u) d u \\
& =\chi\left(c_{1} c_{2}\right)+\int_{\mathbb{R}} e^{i t u}\left(\chi\left(f_{1}\right) \chi\left(c_{2}\right)+\chi\left(c_{1}\right) \chi\left(f_{2}\right)+\chi\left(f_{1}\right) \circ \chi\left(f_{2}\right)\right)(u) d u .
\end{aligned}
$$

Moreover,

$$
\begin{gathered}
\omega\left(F_{1}\right)(i t) \omega\left(F_{2}\right)(i t)=\left(\chi\left(c_{1}\right)+\int_{\mathbb{R}} e^{i t u} \chi\left(f_{1}(u)\right) d u\right)\left(\chi\left(c_{2}\right)+\int_{\mathbb{R}} e^{i t u} \chi\left(f_{2}(u)\right) d u\right) \\
=\chi\left(c_{1} c_{2}\right)+\int_{\mathbb{R}} e^{i t u}\left(\chi\left(f_{1}\right) \chi\left(c_{2}\right)+\chi\left(c_{1}\right) \chi\left(f_{2}\right)+\chi\left(f_{1}\right) \circ \chi\left(f_{2}\right)\right)(u) d u,
\end{gathered}
$$

where the last equality follows from the case of functions in $\mathcal{W}^{2 \times 2}(\mathbb{R})$.

Our main result as follows:

Theorem 4.3. Let $F \in \mathcal{W}(\mathbb{R}, \mathbb{H})$. The following are equivalent:

(i) The function $F$ is invertible in $\mathcal{W}(\mathbb{R}, \mathbb{H})$;

(ii) For any $i \in \mathbb{S}$ we have $\operatorname{det} \omega(F)(i t) \neq 0$, for all $t \in \mathbb{R}$;

(iii) There exists an $i \in \mathbb{S}$ such that $\operatorname{det} \omega(F)(i t) \neq 0$, for all $t \in \mathbb{R}$;

(iv) The function $F(p) \neq 0$ for all $p \in \mathbb{R} \mathbb{S}$. 
Proof. Assume that (i) holds, and denote by $F^{-1}$ the inverse of $F$ in $\mathcal{W}(\mathbb{R}, \mathbb{H})$. Since $\left(F \star F^{-1}\right)(p)=1, p \in \mathbb{S R}$, Lemma 4.2 gives $\omega(F)(i t) \omega\left(F^{-1}\right)(i t)=I_{2}$ and so $\operatorname{det} \omega(F)(i t) \neq 0$ and (ii) holds. To show the converse, let us assume that $\operatorname{det} \omega(F)(i t) \neq 0$, for all $t \in \mathbb{R}$, for some fixed $i \in \mathbb{S}$. Thus the function $\omega(F)(i t)$ admits an inverse in $\mathcal{W}^{2 \times 2}(\mathbb{R})$, where $F$ is as in formula (4.1), given by

$$
\omega(F)^{-1}(i t)=M+\int_{\mathbb{R}} e^{i t u}\left(\begin{array}{cc}
c(u) & d(u) \\
e(u) & f(u)
\end{array}\right) d u .
$$

Since $c_{1} \neq 0$, and so $\chi\left(c_{1}\right)$ is invertible, we have $M=\chi\left(c_{1}^{-1}\right)$. It is not reductive to assume that $M=I_{2}$, (in the general case the computations below are slightly longer but they are essentially the same). By considering the product $\omega(F) \omega(F)^{-1}$, we obtain

$$
\begin{aligned}
& I_{2}=\omega(F)(i t) \omega(F)^{-1}(i t)=I_{2}+\int_{\mathbb{R}} e^{i t u}\left(\int_{\mathbb{R}}\left(\begin{array}{cc}
\frac{a(u-v)}{-\overline{b(u-v)}} & \frac{b(u-v)}{a(u-v)}
\end{array}\right)\left(\begin{array}{cc}
c(v) & d(v) \\
e(v) & f(v)
\end{array}\right) d v+\right. \\
& \left(\begin{array}{ll}
c(u) & d(u) \\
e(u) & f(u)
\end{array}\right)+\left(\begin{array}{cc}
a(u) & \left.\frac{b(u)}{a(u)}\right)
\end{array}\right) d u
\end{aligned}
$$

and so

$$
\begin{aligned}
& \int_{\mathbb{R}} e^{i t u}\left\{\int_{\mathbb{R}}\left(\begin{array}{cc}
a(u-v) & \frac{b(u-v)}{-\overline{b(u-v)}} \\
a(u-v)
\end{array}\right)\left(\begin{array}{ll}
c(v) & d(v) \\
e(v) & f(v)
\end{array}\right) d v+\right. \\
& \left(\begin{array}{ll}
c(u) & d(u) \\
e(u) & f(u)
\end{array}\right)+\left(\begin{array}{cc}
a(u) & \left.\frac{b(u)}{a(u(u)}\right)
\end{array}\right\} d u=0_{2},
\end{aligned}
$$

which gives

$$
\int_{\mathbb{R}}\left(\begin{array}{cc}
a(u-v) & \frac{b(u-v)}{a(u-v)}
\end{array}\right)\left(\begin{array}{ll}
c(v) & d(v) \\
e(v) & f(v)
\end{array}\right) d v+\left(\begin{array}{cc}
c(u) & d(u) \\
e(u) & f(u)
\end{array}\right)+\left(\begin{array}{cc}
a(u) & b(u) \\
-\overline{b(u)} & \left.\frac{a(u)}{a(u)}\right)=0_{2} .
\end{array}\right.
$$

With $J_{1}$ given by (2.8), let us take the conjugate of equation (4.2) and multiply in a suitable way by $J_{1}$ and $J_{1}^{T}$ to obtain

which gives

$$
\begin{aligned}
& \int_{\mathbb{R}} J_{1}\left(\begin{array}{cc}
\overline{a(u-v)} & \overline{b(u-v)} \\
-b(u-v) & a(u-v)
\end{array}\right) J_{1}^{T} J_{1}\left(\overline{\frac{c(v)}{e(v)}} \frac{\overline{d(v)}}{f(v)}\right) J_{1}^{T} d v \\
& +J_{1}\left(\begin{array}{ll}
\overline{\frac{c(v)}{e(v)}} & \overline{d(v)} \\
f(v)
\end{array}\right) J_{1}^{T}+J_{1}\left(\begin{array}{cc}
\overline{a(u)} & \overline{b(u)} \\
-b(u) & a(u)
\end{array}\right) J_{1}^{T}=0_{2}
\end{aligned}
$$

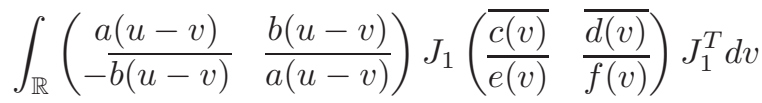

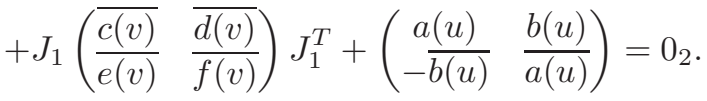

Since equation (4.2) admits a unique solution we deduce that

$$
J_{1}\left(\begin{array}{ll}
\overline{c(v)} & \overline{d(v)} \\
\hline f(v)
\end{array}\right) J_{1}^{T}=\left(\begin{array}{ll}
c(v) & d(v) \\
e(v) & f(v)
\end{array}\right)
$$

from which it follows $e(u)=-\overline{d(u)}$ and $f(u)=\overline{c(u)}$. Thus $\omega(F)^{-1}=\omega(G)$ with

$$
G(i t)=c_{1}^{-1}+\int_{\mathbb{R}} e^{i t u}(c(u)+d(u) j) d u
$$


and $G \in \mathcal{W}(\mathbb{R}, \mathbb{H})$ since $c, d \in L_{1}(\mathbb{R}, \mathbb{H})$. The function $G$ is the inverse of $F$ in $\mathcal{W}(\mathbb{R}, \mathbb{H})$.

It is trivial to show that (ii) implies (iii). We now show that (iii) implies (iv). Let us fix $i$ and rewrite $\omega(F)(i t)$ in the form

$$
\omega(F)(i t)=\left(\begin{array}{ll}
\frac{A(i t)}{-B(-i t)} & \frac{B(i t)}{A(-i t)}
\end{array}\right)
$$

where

$$
A(i t)=\alpha+\int_{\mathbb{R}} e^{i t u} a(u) d u, \quad B(i t)=\beta+\int_{\mathbb{R}} e^{i t u} b(u) d u
$$

where $c_{1}=\alpha+\beta j$.

Suppose to the contrary that there exists $t_{0} \in \mathbb{R}$ such that $F\left(i t_{0}\right)=0$. Then $A\left(i t_{0}\right)=B\left(i t_{0}\right)=0$ and so we have $\operatorname{det}(\omega(F))\left(i t_{0}\right)=0$ a contradiction.

Let us now assume (iv), and prove that (i) is in force. Since $F(i t)$ is invertible for all $t \in \mathbb{R}$ and all $i \in \mathbb{S}$, the matrix $\chi(F)(i t)$ is invertible for every $i \in \mathbb{S}$ and $t \in \mathbb{R}$ and so the function $\chi(F)$ is invertible in $\mathcal{W}^{2 \times 2}(\mathbb{R})$ for every $i \in \mathbb{S}$. Let us write

$$
\begin{aligned}
(\chi(F))(i t) & =\chi\left(c_{1}\right)+\left(\begin{array}{cc}
\int_{\mathbb{R}} e^{i t u} a(u) d u & \int_{\mathbb{R}} e^{i t u} b(u) d u \\
-\int_{\mathbb{R}} e^{-i t u} \overline{b(u)} d u & \int_{\mathbb{R}} e^{-i t u} \overline{a(u)} d u
\end{array}\right) \\
& =\chi\left(c_{1}\right)+\left(\begin{array}{cc}
\int_{\mathbb{R}} e^{i t u} a(u) d u & \int_{\mathbb{R}} e^{i t u} b(u) d u \\
-\int_{\mathbb{R}} e^{i t u} \overline{b(-u)} d u & \int_{\mathbb{R}} e^{i t u} \overline{a(-u)} d u
\end{array}\right)
\end{aligned}
$$

and since $\chi(F)$ is invertible in $\mathcal{W}^{2 \times 2}(\mathbb{R})$, there exists $H$ such that $(\chi(F)(i t)) H(i t)=$ $I_{2}$. Let us write

$$
H(i t)=\chi\left(c_{1}^{-1}\right)+\left(\begin{array}{ll}
C(i t) & D(i t) \\
E(i t) & L(i t)
\end{array}\right)
$$

and, up to a normalization we assume that $\chi\left(c_{1}\right)=\chi\left(c_{1}^{-1}\right)=I_{2}$. Then we have

$$
\begin{aligned}
\chi(F)(i t) H(i t) & =I_{2} \\
& =\left(I_{2}+\left(\begin{array}{cc}
\int_{\mathbb{R}} e^{i t u} a(u) d u & \int_{\mathbb{R}} e^{i t u} b(u) d u \\
-\int_{\mathbb{R}} e^{i t u} \overline{b(-u)} d u & \int_{\mathbb{R}} e^{i t u} \overline{a(-u)} d u
\end{array}\right)\left(I_{2}+\left(\begin{array}{cc}
C(i t) & D(i t) \\
E(i t) & L(i t)
\end{array}\right)\right)\right. \\
& =I_{2}+\left(\begin{array}{cc}
\int_{\mathbb{R}} e^{i t u} a(u) d u & \int_{\mathbb{R}} e^{i t u} b(u) d u \\
-\int_{\mathbb{R}} e^{i t u} \overline{b(-u)} d u & \int_{\mathbb{R}} e^{i t u} \overline{a(-u)} d u
\end{array}\right)\left(\begin{array}{cc}
C(i t) & D(i t) \\
E(i t) & L(i t)
\end{array}\right) \\
& +\left(\begin{array}{cc}
\int_{\mathbb{R}} e^{i t u} a(u) d u & \int_{\mathbb{R}} e^{i t u} b(u) d u \\
-\int_{\mathbb{R}} e^{i t u} \overline{b(-u)} d u & \int_{\mathbb{R}} e^{i t u} \overline{a(-u)} d u
\end{array}\right)+\left(\begin{array}{cc}
C(i t) & D(i t) \\
E(i t) & L(i t)
\end{array}\right)
\end{aligned}
$$


is uniquely solvable. Reasoning as in the proof of (ii) implies (i), we can take the conjugate of (4.3) and suitably multiplying by $J_{1}$ and $J_{1}^{T}$ we obtain:

$$
\begin{aligned}
& I_{2}=I_{2}+J_{1}\left(\begin{array}{cc}
\int_{\mathbb{R}} e^{-i t u} \overline{a(u)} d u & \int_{\mathbb{R}} e^{-i t u} \overline{b(u)} d u \\
-\int_{\mathbb{R}} e^{-i t u} b(-u) d u & \int_{\mathbb{R}} e^{-i t u} a(-u) d u
\end{array}\right) J_{1}^{T} J_{1}\left(\overline{\frac{C(i t)}{E(i t)}} \overline{\overline{D(i t)}} \overline{L(i t)}\right) J_{1}^{T} \\
& +J_{1}\left(\begin{array}{cc}
\int_{\mathbb{R}} e^{-i t u} \overline{a(u)} d u & \int_{\mathbb{R}} e^{-i t u} \overline{b(u)} d u \\
-\int_{\mathbb{R}} e^{-i t u} b(-u) d u & \int_{\mathbb{R}} e^{-i t u} a(-u) d u
\end{array}\right) J_{1}^{T}+J_{1}\left(\begin{array}{ll}
\overline{C(i t)} & \overline{D(i t)} \\
E(i t) & \overline{L(i t)}
\end{array}\right) J_{1}^{T} \\
& =I_{2}+\left(\begin{array}{cc}
\int_{\mathbb{R}} e^{-i t u} a(-u) d u & \int_{\mathbb{R}} e^{-i t u} b(-u) d u \\
-\int_{\mathbb{R}} e^{-i t u} \overline{b(u)} d u & \int_{\mathbb{R}} e^{-i t u} \overline{a(u)} d u
\end{array}\right)\left(\begin{array}{cc}
\overline{L(i t)} & -\overline{E(i t)} \\
-\overline{D(i t)} & \overline{C(i t)}
\end{array}\right) \\
& +\left(\begin{array}{ll}
\int_{\mathbb{R}} e^{-i t u} a(-u) d u & \int_{\mathbb{R}} e^{-i t u} b(-u) d u \\
-\int_{\mathbb{R}} e^{-i t u} \overline{b(u)} d u & \int_{\mathbb{R}} e^{-i t u} \overline{a(u)} d u
\end{array}\right)+\left(\begin{array}{cc}
\overline{L(i t)} & -\overline{E(i t)} \\
-\overline{D(i t)} & \overline{C(i t)}
\end{array}\right) \\
& =I_{2}+\left(\begin{array}{cc}
\int_{\mathbb{R}} e^{i t u} a(u) d u & \int_{\mathbb{R}} e^{i t u} b(u) d u \\
-\int_{\mathbb{R}} e^{i t u} \overline{b(-u)} d u & \int_{\mathbb{R}} e^{i t u} \overline{a(-u)} d u
\end{array}\right)\left(\begin{array}{cc}
\overline{L(i t)} & -\overline{E(i t)} \\
-\overline{D(i t)} & \overline{C(i t)}
\end{array}\right) \\
& +\left(\begin{array}{cc}
\int_{\mathbb{R}} e^{i t u} a(u) d u & \int_{\mathbb{R}} e^{i t u} b(u) d u \\
-\int_{\mathbb{R}} e^{i t u} \overline{b(-u)} d u & \int_{\mathbb{R}} e^{i t u} \overline{a(-u)} d u
\end{array}\right)+\left(\begin{array}{cc}
\overline{L(i t)} & -\overline{E(i t)} \\
-\overline{D(i t)} & \overline{C(i t)}
\end{array}\right) .
\end{aligned}
$$

We deduce that $C(i t)=\overline{L(i t)}, D(i t)=-\overline{E(i t)}, E(i t)=-\overline{D(i t)}, L(i t)=\overline{C(i t)}$ and

$$
H(i t)=I_{2}+\left(\begin{array}{cc}
\frac{C(i t)}{D(i t)} & \frac{D(i t)}{C(i t)}
\end{array}\right)
$$

The function $1+(C(i t)+D(i t) j)=1+\int_{\mathbb{R}} e^{i t u}(c(u)+d(u) j) d u$, for some suitable $c(u), d(u)$ is, by construction the inverse of $F$ in $\mathcal{W}(\mathbb{R}, \mathbb{H})$ and thus (i) holds. This concludes the proof.

Unlike in the discrete case, the notion of positivity and the associated factorization is slice dependent. To illustrate this fact, fix some $i \in \mathbb{S}$ and consider the function $F(p)=\frac{1-i p}{1+p^{8}}$. Then $F(i t)=\frac{1+t}{1+t^{8}}$ belongs to the classical Wiener algebra (since it is real valued and has no no poles on the real line; see for instance [2]) and there exists $M>0$ such that $G(t)=M+F(i t)>0$ for all $t \in \mathbb{R}$. So the function $G(t)$ admits a spectral factorization. On the other hand for $j \in \mathbb{S}$ different from $\pm i$ the function $F(j t)$ is not real valued, and, in particular, is not positive on the real line.

Definition 4.4. Fix $i \in \mathbb{S}$. We denote by $\mathcal{W}_{+}(i \mathbb{R}, \mathbb{H})$ and $\mathcal{W}_{-}(i \mathbb{R}, \mathbb{H})$ the set of elements $F(i y)=c+\int_{-\infty}^{\infty} e^{i y u} f(u) d u$, with $\left.F \in \mathcal{W}(\mathbb{R}, \mathbb{H})\right)$ so that $f(u)=0$ for $u<0$ and $f(u)=0$ for $u>0$, respectively.

It is immediate that $\mathcal{W}_{+}(i \mathbb{R}, \mathbb{H})$ and $\mathcal{W}_{-}(i \mathbb{R}, \mathbb{H})$ are subalgebras of $\mathcal{W}(\mathbb{R}, \mathbb{H})$.

Theorem 4.5. Let $F \in \mathcal{W}_{+}(i \mathbb{R}, \mathbb{H})$. Then the following are equivalent:

(i) $F$ is invertible in $\mathcal{W}_{+}(i \mathbb{R}, \mathbb{H})$ (with inverse denoted by $F^{-1}$ );

(ii) $\omega(F)$ is invertible in $\mathcal{W}_{+}^{2 \times 2}(\mathbb{R})$.

Proof. Assume that (i) holds. Then $\omega(F)(i t) \omega\left(F^{-1}\right)(i t)=I_{2}$. Since both functions $\omega(F)(i t)$ and $\omega\left(F^{-1}\right)(i t)$ have analytic extensions (as functions of $t$ ) to the open left half-plane, (ii) follows.

Assume now that (ii) holds. Then the same arguments as in the proof of Theorem 4.3 (with integrals on $[0, \infty)$ instead of $\mathbb{R}$ ) will show that $(i)$ holds.

We note that if $F \in \mathcal{W}(\mathbb{R}, \mathbb{H})$ is strictly positive on $i \mathbb{R}$ it admits a spectral factorization with factor in $\mathcal{W}_{+}(i \mathbb{R}, \mathbb{H})$. The arguments are similar and omitted. 


\section{HARDY SPACES AND WiENER-Hopf OPERATORS}

We will begin by recalling some classical facts from the theory of Hardy spaces. Let $i \in \mathbb{S}$. We shall identify as usual the complex plane $\mathbb{C}_{i}$ with $\mathbb{C}$. Let $\Pi_{+}=\{z \in \mathbb{C}$ : Re $z>0\}$. The Hardy space $H_{2}\left(\Pi_{+}\right)$consists of functions $f$ which are holomorphic on $\Pi_{+}$and satisfy

$$
\sup _{x>0} \int_{-\infty}^{\infty}|f(x+i y)|^{2} d y<\infty .
$$

Recall that $f \in H_{2}\left(\Pi_{+}\right)$has nontangential limits $f(i y)$ for almost all (with respect to the Lebesgue measure) $i y,\left.f\right|_{i \mathbb{R}} \in L_{2}(\mathbb{R})$ and

$$
\sup _{x>0} \int_{-\infty}^{\infty}|f(x+i y)|^{2} d y=\int_{-\infty}^{\infty}|f(i y)|^{2} d y .
$$

An important fact is that $f \in H_{2}\left(\Pi_{+}\right)$if and only if there exists $\tilde{f} \in L_{2}(\mathbb{R})$ such that

$$
f(z)=\int_{0}^{\infty} e^{i z x} \tilde{f}(x) d x \quad \text { for } \quad z \in \Pi_{+} .
$$

Upon identifying functions $f \in H_{2}\left(\Pi_{+}\right)$with their boundary values, it can be checked that $H_{2}\left(\Pi_{+}\right)$is a closed subspace of $L_{2}(i \mathbb{R})$, i.e., the Hilbert space of complex-valued functions $f$ defined on $i \mathbb{R}$ so that

$$
\int_{-\infty}^{\infty}|f(i y)|^{2} d y<\infty
$$

The inner product $\langle\cdot, \cdot\rangle_{L_{2}(i \mathbb{R})}$ is given by

$$
\langle f, g\rangle_{L_{2}(i \mathbb{R})}=\int_{-\infty}^{\infty} \overline{g(i y)} f(i y) d y .
$$

It can also be checked that $H_{2}\left(\Pi_{-}\right):=H_{2}\left(\Pi_{+}\right)^{\perp}$ consists of functions $f$ which are holomorphic on $\Pi_{-}=\{z \in \mathbb{C}: \operatorname{Re} z<0\}$ and satisfy

$$
\sup _{x<0} \int_{-\infty}^{\infty}|f(x+i y)|^{2} d y<\infty .
$$

An important fact is that $f \in H_{2}\left(\Pi_{-}\right)$if and only if there exists $\tilde{f} \in L_{2}(\mathbb{R})$ such that

$$
f(z)=\int_{-\infty}^{0} e^{i z x} \tilde{f}(x) d x .
$$

Finally, upon identifying functions $H_{2}\left(\Pi_{+}\right)$and $H_{2}\left(\Pi_{-}\right)$via their boundary values with functions in $L_{2}(i \mathbb{R})$,

$$
L_{2}(i \mathbb{R})=H_{2}\left(\Pi_{+}\right) \oplus H_{2}\left(\Pi_{-}\right)
$$

follows from (5.1) and (5.2).

Returning to the quaternionic setting, see [1], let $\mathbb{H}_{+}=\{p \in \mathbb{H}: \operatorname{Re} p>0\}$ and $\Pi_{+, i}=\mathbb{H}_{+} \cap \mathbb{C}_{i}$ for any $i \in \mathbb{S}$. Let $f_{i}=\left.f\right|_{\Pi_{+, i}}$, where $f$ is a function defined on $\mathbb{H}_{+}$. We let $H_{2}\left(\Pi_{+}, i\right)$ denote the space of slice hyperholomorphic functions on $\mathbb{H}_{+}$ so that

$$
\int_{-\infty}^{\infty}\left|f_{i}(i y)\right|^{2} d y<\infty
$$

where $f_{i}(i y)$ denote the nontangential limit at $i y$ of $f_{i}$. 
Given $f \in H_{2}\left(\Pi_{+, i}\right)$, the restriction $f_{i}$ can be written as

$$
f_{i}(x+i y)=f_{1}(x+i y)+f_{2}(x+i y) j,
$$

where $j \in \mathbb{S}$ so that $i j=-1$, i.e., $i$ and $j$ are orthogonal and $f_{1}, f_{2}$ are $\mathbb{C}_{i}$-valued holomorphic functions. Note that

$$
f_{i}(i y)=f_{1}(i y)+f_{2}(i y) j \quad \text { a.e. }
$$

and

$$
\left|f_{i}(x+i y)\right|^{2}=\left|f_{1}(x+i y)\right|^{2}+\left|f_{2}(x+i y)\right|^{2} .
$$

Thus, making use of (5.5) and (5.6) we get

$$
\begin{aligned}
\sup _{x>0} \int_{-\infty}^{\infty}\left|f_{i}(x+i y)\right|^{2} d y & =\int_{-\infty}^{\infty}\left|f_{1}(i y)\right|^{2} d y+\int_{-\infty}^{\infty}\left|f_{2}(i y)\right|^{2} d y \\
& =\int_{-\infty}^{\infty}\left|f_{i}(i y)\right|^{2} d y .
\end{aligned}
$$

Let $j \in \mathbb{S}$ be orthogonal to $i \in \mathbb{S}$. We let $L_{2}(i \mathbb{R}, \mathbb{H})$ consist of all functions $g$ of the form

$$
g(i y)=g_{1}(i y)+g_{2}(i y) j
$$

where $g_{1}$ and $g_{2}$ belong to $L_{2}(i \mathbb{R})$, with norm

$$
\|g\|_{L_{2}(i \mathbb{R}, \mathbb{H})}=\left(\left\|g_{1}\right\|_{L_{2}(i \mathbb{R})}^{2}+\left\|g_{2}\right\|_{L_{2}(i \mathbb{R})}^{2}\right)^{1 / 2}
$$

Summarizing the above discussion we arrive at Theorem 5.1 and 5.2

Theorem 5.1. Let $j \in \mathbb{S}$ be orthogonal to $i \in \mathbb{S}$. The space $H_{2}\left(\Pi_{+, i}\right)$ is a closed subspace of $L_{2}(i \mathbb{R}, \mathbb{H})$, upon identifying functions in $H_{2}\left(\Pi_{+, i}\right)$ with their boundary values. Moreover,

$$
L_{2}(i \mathbb{R}, \mathbb{H})=H_{2}\left(\Pi_{+, i}\right) \oplus H_{2}\left(\Pi_{+, i}\right)^{\perp} .
$$

Theorem 5.2. Fix $i \in \mathbb{S}$. A function $f \in H_{2}\left(\Pi_{+, i}\right)$ if and only if $f_{1} \in H_{2}\left(\Pi_{+}\right)$ and $f_{2} \in H_{2}\left(\Pi_{+}\right)$, where $f_{1}$ and $f_{2}$ are as in (5.4).

Corollary 5.3. Fix $i \in \mathbb{S}$. A function $f \in H_{2}\left(\Pi_{+, i}\right)$ if and only if

$$
f_{i}(x+i y)=\int_{0}^{\infty} e^{i(x+i y) u} \tilde{f}_{1}(u) d u+\left(\int_{0}^{\infty} e^{i(x+i y) u} \tilde{f}_{2}(u) d u\right) j,
$$

where $x>0, \tilde{f}_{1}$ and $\tilde{f}_{2}$ belong to $L_{2}(\mathbb{R})$ and $j \in \mathbb{S}$ is orthogonal to $i \in \mathbb{S}$.

Proof. Assertion (5.9) follows easily from Theorem 5.2 and (5.1).

With these definitions and results at hand we turn to an application to the theory of Wiener-Hopf operators. Fix $i \in \mathbb{S}$ and let $j \in \mathbb{S}$ be orthogonal to $i$, i.e., $i j=$ -1 . Let $P_{i}$ and $Q_{i}$ denote the orthogonal projections of $L_{2}(i \mathbb{R}, \mathbb{H})$ onto $H_{2}\left(\Pi_{+, i}\right)$ and $H_{2}\left(\Pi_{+, i}\right)^{\perp}$. Note that $P_{i}$ and $Q_{i}$ are well-defined due to Theorem [5.1] If $\Phi \in \mathcal{W}(\mathbb{R}, \mathbb{H})$ and $F \in H_{2}\left(\Pi_{+, i}\right)$, then there exist a constant $c_{1}, \phi \in L_{1}(\mathbb{R})$ and $f \in L_{2}(i \mathbb{R})$ so that

$$
\Phi(i y)=c_{1}+\int_{-\infty}^{\infty} e^{i y u} \phi(u) d u
$$

and

$$
F(i y)=\int_{0}^{\infty} e^{i y u} f(i u) d u \quad \text { a.e.. }
$$


Thus, we can define

$$
(\Phi \star F)(i y)=c_{1} \int_{0}^{\infty} e^{i y u} f(u) d u+\int_{-\infty}^{\infty} e^{i y u}(\phi \circ f)(u) d u
$$

where

$$
(\phi \circ f)(u)=\int_{-\infty}^{\infty} \phi(u-v) f(v) d v .
$$

Since $F \in H_{2}\left(\Pi_{+, i}\right) \in H_{2}\left(\Pi_{+, i}\right) \subset L_{2}(i \mathbb{R}, \mathbb{H})$, we have that $\Phi \star F \in L_{2}(i \mathbb{R}, \mathbb{H})$ and hence

$$
M_{\Phi} F:=\Phi \star F
$$

defines a multiplication operator from $H_{2}\left(\Pi_{+, i}\right)$ to $L_{2}(i \mathbb{R}, \mathbb{H})$.

We are now ready to define the Wiener-Hopf operator based on $\Phi \in \mathcal{W}(\mathbb{R}, \mathbb{H})$. The operator $T_{\Phi}: H_{2}\left(\Pi_{+, i}\right) \rightarrow H_{2}\left(\Pi_{+, i}\right)$ given by

$$
T_{\Phi} F=P_{i} M_{\Phi} F \quad \text { for } \quad F \in H_{2}\left(\Pi_{+, i}\right)
$$

is the Wiener-Hopf operator based on $\Phi \in \mathcal{W}(\mathbb{R}, \mathbb{H})$.

Theorem 5.4. Fix $i \in \mathbb{S}$. If $\Phi, \Psi \in \mathcal{W}(\mathbb{R}, \mathbb{H})$, then $T_{\Phi} T_{\Psi}$ is a Wiener-Hopf operator if and only if $\Phi \in \mathcal{W}_{-}(i \mathbb{R}, \mathbb{H})$ or $\Psi \in \mathcal{W}_{+}(i \mathbb{R}, \mathbb{H})$. In this case, $T_{\Phi} T_{\Psi}=$ $T_{\Phi \star \Psi}$.

Proof. Theorem 5.4 is proved in much the same way as Theorem 3.1 .

\section{REFERENCES}

[1] D. Alpay, F. Colombo, I. Lewkowicz and I. Sabadini. Realizations of slice hyperholomorphic generalized contractive and positive functions, ArXiv e-prints, 2013, to appear in Milan J. Math.

[2] H. Bart, I. Gohberg and M.A. Kaashoek. Convolution equations and linear systems. Integral Equations Operator Theory 5 (1982), no. 3, 283340.

[3] S. Bochner and R. S. Phillips. Absolutely convergent Fourier expansions for non-commutative normed rings. Ann. of Math. 43, (1942), 409-418.

[4] A. Brown and P. Halmos. Algebraic properties of Toeplitz operators, J. Reine Angew. Math. 213 1963/1964 89-102.

[5] F. Colombo, I. Sabadini and D. C. Struppa. Noncommutative functional calculus. Theory and applications of slice hyperholomorphic functions, volume 289 of Progress in Mathematics. Birkhäuser/Springer Basel AG, Basel, 2011.

[6] H. Dym and I. Gohberg. Extensions of matrix valued functions with rational polynomial inverses, Integral Equations Operator Theory, 2 (1979), 503-528.

[7] H. Dym and I. Gohberg. Extension of matrix valued functions and block matrices, Indiana Univ. Math. J., 31 (1982), 733-765.

[8] M. Fliess. Matrices de Hankel. J. Math. Pures Appl. (9), 53:197-222, 1974.

[9] I. Gohberg, S. Goldberg and M. A. Kaashoek. Classes of linear operators. Vol. II. Operator Theory: Advances and Applications, 63. Birkhäuser Verlag, Basel, 1993.

[10] G. Gentili, C Stoppato and D.C. Struppa. Regular Functions of a Quaternionic Variable, Springer Monographs in Mathematics, Springer, Berlin-Heidelberg (2013).

[11] T. Y. Lam. A First Course in Noncommutative Rings, Graduate Texts in Mathematics, 123, Springer-Verlag, New York, (1991), 261-263.

[12] W. Rudin. Real and complex analysis, McGraw-Hill Book Co., 1987.

[13] N. Wiener. Generalized harmonic analysis, Acta Math., 55 (1930), 117-258. 
(DA) Department of Mathematics, Ben-Gurion University of the Negev, Beer-Sheva 84105 ISRAEL

E-mail address: dany@math.bgu.ac.il

(FC) Politecnico di Milano, Dipartimento di Matematica, Via E. Bonardi, 9, 20133 MiLANO, ITALY

E-mail address: fabrizio.colombo@polimi.it

(DPK) Department of Mathematics, Ben-Gurion University of the Negev, Beer-Sheva 84105 ISRAEL

E-mail address: dpkimsey@gmail.com

(IS) Politecnico di Milano, Dipartimento di Matematica, Via E. Bonardi, 9, 20133 MiLANO, ITALY

E-mail address: irene.sabadini@polimi.it 